\title{
Preface to SeCoGIS 2011
}

This volume contains the papers presented at SeCoGIS 2011, the Fifth International Workshop on Semantic and Conceptual Issues in GIS, held the 1st of November in Brussels, Belgium.

Current information technologies have increased the production, collection, and diffusion of geographical and temporal data, thus favoring the design and development of geographic information systems (GIS) and more generally speaking spatio-temporal information systems (STIS). Nowadays, GISs are emerging as a common information infrastructure, which penetrate into more and more aspects of our society. This has given rise to new methodological and data engineering challenges in order to accommodate new users' requirements for new applications. Conceptual and semantic modeling are ideal candidates to contribute to the development of the next generation of GIS solutions. They allow eliciting and capturing user requirements as well as the semantics of a wide domain of applications.

The SeCoGIS workshop brings together researchers, developers, users, and practitioners carrying out research and development in geographic information systems. The aim is to stimulate discussions on the integration of conceptual modeling and semantics into current geographic information systems, and how this will benefit the end users. The workshop provides a forum for original research contributions and practical experiences of conceptual modeling and semantic web technologies for GIS, fostering interdisciplinary discussions in all aspects of these two fields, and will highlight future trends in this area. The workshop is organized in a way to highly stimulate interaction amongst the participants.

This edition of the workshop attracted papers from 15 different countries distributed all over the world: Brazil, Argentina, Chile, USA, Canada, France, Italy, Spain, Germany, Belgium, The Netherlands, Austria, Macedonia FYROM, Morocco, and New-Zealand. We received 19 papers from which the Program Committee selected 6 papers, making an acceptance rate of 32 percent. The accepted papers cover a wide range of issues, in particular consistency and integration, refined spatial relationships and conceptual model transformation rules.

We hope that you find the program and presentations beneficial and enjoyable and that during the workshop you had many opportunities to meet colleagues and practitioners. We would like to express our gratitude to the program committee members and the external referees for their hard work in reviewing papers, the authors for submitting their papers, and the ER 2011 organizing committee for all their support.

July, 2011

Esteban Zimányi

Roland Billen

Pierre Hallot 